\section{ONGOING INHIBITION OF BACTERIAL COLONISATION}

Beautifil II Gionomer from Shofu is a state of the art composite restorative material based on Pre-Reacted Glass Ionomer technology. This technology combines the benefits of glass ionomers with those of composite resins to produce a biocompatible, fluoride releasing, high strength, beautifully aesthetic material.

Due to the ongoing release and recharge of the fluoride particles within Beautifil II, secondary caries are no longer a concern, with the added benefit that plaque will not stick to the surface of a Beautifil II restoration. Saliva forms a material film on the surface of Beautifil II that is reported to minimise plaque adhesion and inhibit bacterial colonisation as an ongoing process.

The material takes on the colour of the

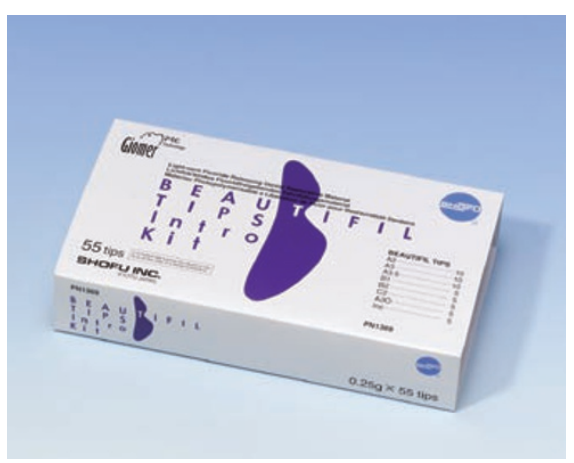

surrounding tooth tissue with a chameleon effect. With a highly translucent finish this radiopaque versatile, highly dependable material exhibits excellent wear resistance combined with a high filler load to ensure longevity of the final restoration.

Reader response number 54

\title{
PROTECT YOUR PRACTICE FROM SWINE FLU
}

The Dental Directory has a range of infection prevention products that will not only help you follow best practice guidelines but will protect you and your patients against the spread of the H1N1 'Swine Flu' virus.

The UnoDent FFP3D valved respirator mask (CHI 015) offers 99\% particle filtration efficiency for those in contact with affected persons. The masks come in boxes of ten and are effective against H1N1.

UnoDent and Classic also offer a range of wipes and refills for hard surfaces and surface disinfection. The UnoDent UnoWipes (CAW 565) and Classic Hard Surface Wipes (CAW 580/585) are effective against H1N1.

The UnoDent and Classic ranges also

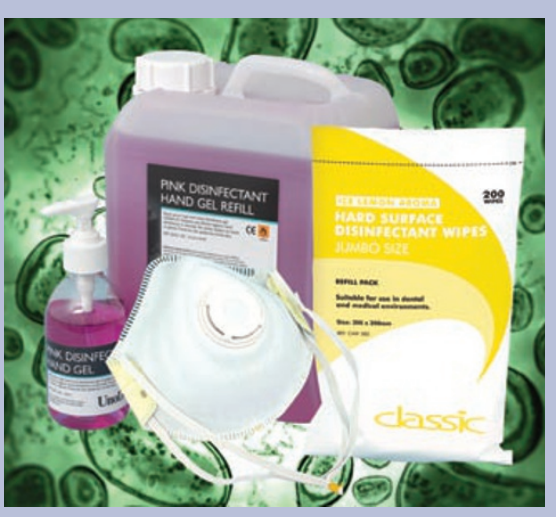

have an extensive selection of gloves, hand disinfectant gels and cleansers, instrument cleaners and disinfectant sprays are also all available - all to prevent the spread of infection in your practice.

Reader response number 55

\section{COMPUTING PERFECT RESTORATIONS}

The CEREC CAD/CAM System from Sirona is proven to increase profits and it also adds the 'wow' factor to any dental practice. CEREC enables you to provide your patients with same day prostheses such as crowns and bridges without the expense and time of waiting for the laboratory to construct them.

Patients are willing to pay that little bit more for one of the most aesthetic, perfectly fitting ceramic restorations in the marketplace today. The finished prosthesis also exhibits excellent strengths with superb margins. CEREC accurately computes the occlusal contacts saving you from making any further adjustments.

Reader response number 56

\section{TURBINE BUILT TO LAST}

The Bora Blackline turbine is new to Bien-Air. With its carbon fibre handle this super-light handpiece offers improved resistance to wear, friction, torsion and impacts. The light weight makes it exceptionally easy to manoeuvre with significantly less hand fatigue for the practitioner.

LED lighting is standard with all Blackline turbines offering unsurpassed visibility in the operative field.

The Bora is incredibly quiet due to ceramic ball bearings. It is also built to last.

Bora Blackline and Metaline turbines are fitted with an integral nonreturn valve to help with infection control and large push buttons for easy bur removal. The turbines also incorporate an anti-heat system to avoid heat build-up during treatment which may cause patient discomfort. The triple spray diffuser with three separate air and water ports ensures optimum cooling even at high speeds. The Unifix quick-connect coupling means that no time is wasted when changing your handpieces.

Reader response number 57

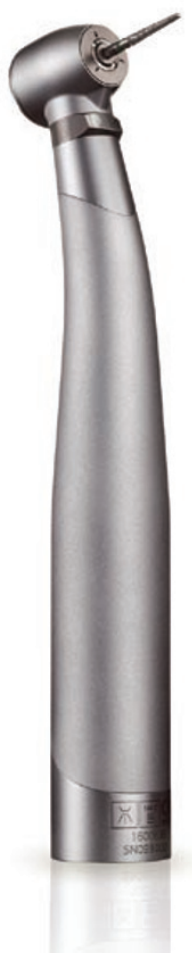

